view of the fact that repeated restrain was required, he was in seclusion and possibility of need for rapid tranquilization post admission the decision was made to use the mental health act.

Conclusion. This case has highlighted a significant problem and calls for an urgent action to increase the number of inpatient age appropriate mental health beds and number of appropriate residential placements nationally. It has also been identified that application of legal frame work in children and adolescents can be a challenge and there is a need for targeted educational programmes for professionals on the use of legal frame work in children and adolescents.

\section{Prolong psychosis preceding cognitive and motor symptoms; an unusual presentation in Huntington's disease}

Nida Khan* and Marium Mansoor

The Aga Khan University Hospital

${ }^{*}$ Corresponding author.

doi: 10.1192/bjo.2021.341

Objective. To describe an unusual clinical presentation of Huntington's disease.

Case report. A 39-years-old married female, a homemaker, presented to the psychiatry clinic with her husband, with over a nineyear history of decreased sleep, suspiciousness, self-talking, agitation, anger outbursts, aggression, and social isolation. The patient was diagnosed with schizophrenia. Previously, she received various antipsychotics and Electroconvulsive therapy (ECT). The patient showed a partial response to treatment. Over the last 2-3 years, the patient had a progressive decline and later required supervision in her Activities of Daily Living (ADLs). She developed slurred speech limited to 1-2 worded answers, gait disturbance, falls, involuntary movements of the trunk and distal extremities, bowel and bladder incontinence, and severe weight loss. The patient's mother and older brother had a history of death in their early 40 s due to an unknown cause.

At presentation, the patient was restless, irritable, self-talking incoherently, neither made nor maintained eye contact and tried hitting and biting upon approaching closely. She did not respond to any queries or followed commands. The patient showed poor personal hygiene. On examination, the patient was hemodynamically stable, had a loss of muscle bulk, broad-based gait, and choreiform movements of the trunk and distal extremities. We admitted the patient to the psychiatry ward and also consulted the neurology team. Her blood investigations showed ASMA antibodies positive, MRI brain was suggestive of Huntington's disease (HD), and her genetic test for Huntington gene confirmed the diagnosis of HD. We started the patient on Fluoxetine, Clonazepam, and Olanzapine. The patient showed a decrease in agitation, and her self-talking stopped.

Discussion. HD is a rare genetic disease that has wellcharacterized symptoms. However, as seen in our patient, these symptoms can evolve and progress unusually in the early and middle stages. Psychosis in HD patients is rare but known. Psychosis is rare in HD and usually presents after a clear clinical picture of HD is apparent. Our case discussed psychotic symptoms in the pre-choreic stage of HD which adds to the existing evidence on challenging presentations and management of HD. Further research can help increase confidence in these outcomes and treatment guidelines.

Conclusion. Our case highlights an unusual clinical presentation of $\mathrm{HD}$, which can be challenging and lead to diagnostic delays.
We recommend a thorough approach to history and revision of diagnosis in case of atypical presentations.

\section{Psychosis in youth in Singapore: a case series}

Pei Ling Lim ${ }^{1 \star}$, Roselyne Shirley ${ }^{2}$ and Pat Fong ${ }^{1}$

${ }^{1}$ Tan Tock Seng Hospital and ${ }^{2}$ KK Women's and Children's Hospital ${ }^{*}$ Corresponding author.

doi: 10.1192/bjo.2021.342

Objective. In this report, we present a case series of children with psychotic symptoms referred to a child consultation liaison psychiatric service within a tertiary paediatric hospital in Singapore. The purpose of this case series is to identify common symptoms at presentation, review the current practices in our hospital for investigation and treatment of first episode psychosis and short-term outcomes.

Case report. We identified 9 cases over a 1 year period, for which 7 were seen whilst admitted to hospital and 2 in the outpatient clinic. There were 6 females and 5 males ranging in age from 11 to 16 years old. The commonest symptoms on presentation were perceptual disturbance (88\%) most commonly auditory hallucinations and altered behaviour (55\%). Of the 7 children admitted to hospital, all were seen by the neurology team prior to the request for a psychiatric opinion. All admitted patients had blood and radiological investigations carried out. Most of the children were started on a short course of antipsychotic medication with the majority continuing to attend follow-up outpatient.

Discussion. Only 9 cases were identified in this case series over a 1 year period highlighting that psychosis is not a common presentation in the paediatric population. From the history alone, it can be challenging to distinguish between primary and secondary causes of psychosis. Acute onset of symptoms and the presence of other neurological signs should raise the suspicion of an underlying organic cause. Out of 9 cases, only 1 case was treated for a presumed organic aetiology, which is consistent with findings from other authors who only found underlying organic factors in $12.5 \%$ of cases.

In this case series, we also noted that $45 \%$ of cases reported having symptoms for over 1 year before seeking help. This is also seen in the adult population in Singapore. Stigma, denial and lack of information about psychosis may all contribute to delay in seeking help. Although prolonged duration of untreated psychosis has been shown to be associated with poor long-term outcome, we found in our case series that even patients who reported a long duration of symptoms still responded well to medication.

Conclusion. There is room for collaboration with our neurology colleagues in the approach towards children with first presentation of psychosis, both in terms of investigations and management. Identifying reasons for disengagement from psychiatric care is an area for further investigations to improve outcomes in our patients.

\section{Management of inappropriate sexual behaviour in frontotemporal dementia: a case study}

Stefan McKenzie*, Zoe Kwan and Velusamy Sivakumar

Sheffield Health and Social Care NHS Foundation Trust ${ }^{\star}$ Corresponding author.

doi: 10.1192/bjo.2021.343 
Objective. To present a case of a 79-year-old male with frontal lobe dementia (following a cerebral abscess) who was referred due to inappropriate sexualised behaviour (ISB) in a care home setting.

To discuss the evidence base for the management of ISB in frontotemporal dementia.

Case report. 79-year-old male patient who was diagnosed with frontal lobe dementia, following a craniotomy to aspirate and evacuate a cerebral abscess which affected the left frontal, parietal and temporal lobes. He then started to exhibit sexualised behaviour; he was using sexualised language towards female residents and care workers in the residential home, and was inviting residents to his room and asking them to touch him. This behaviour was felt to be due to inappropriate sexual behaviour which forms part of the spectrum of behavioural and psychological symptoms of dementia. Non-pharmacological interventions were tried but failed to manage his symptoms. He was started on Paroxetine which treated the symptoms for approximately 12 months. The symptoms reocurred and he was switched to Amisulpride which had a positive effect on his symptoms.

Discussion. ISB is a behavioural and psychological symptom of dementia and may be seen in $7 \%$ to $25 \%$ of patients with dementia. ISB is distressing for the caregivers and also presents considerable challenges for the treating clinician. ISB presents with behaviour such as sexual language, implied sexual acts, and overt sexual acts. A differentiation should be made between whether the act was one of intimacy-seeking or disinhibition. However, there is a need to intervene when there are risks to the wellbeing and safeguards of the patient and also caregivers and residents. ISB can be difficult to treat, and there is limited evidence on the subject. It is often better managed by non-pharmacological interventions if possible, due to patients often being less responsive to psychoactive therapies and the risks involved with using medication. Non-pharmacological interventions include environmental, behavioural and educational approaches, and examples of these are discussed. Pharmacological interventions are also discussed, but there is a lack of evidence in this area; currently the evidence is from case series and case reports. The variety of drug classes illustrate the non specific nature of drug therapy.

Conclusion. Managing and treating ISB is difficult and complex.

The evidence suggests using non-pharmacological approaches as first line before considering pharmacological interventions.

However, there is a need for further research to develop robust non-pharmacological and pharmacological interventions in the treatment of ISB.

A rare case of a patient with resistant schizophrenia who hears a voice reading the texts instead of being read in her mind

\author{
Thilini Nanayakkara* and Vipula Wijesiri \\ Teaching Hospital Peradeniya \\ ${ }^{\star}$ Corresponding author.
}

doi: 10.1192/bjo.2021.344

Objective. This case is presented to describe a rare psychopathology in which the patient hears her own voice speaking out loud all the texts that she sees in books or papers and she cannot read them inside her mind. This psychopathological phenomena has some features of reflex hallucinations, thought echo as well as of inner reading voices yet it cannot be categorized into either one.

Case report. This is a 26-year-old female with Schizophrenia for 3 years. While on medication 8 months before presentation she started hearing her own voice reading any text that she sees. When she sees a text she cannot read it in her mind and understand, but she hears it in her own voice to her ears. With this she also hears other voices talking about her and to her. She also believes that her father is the one who controls all her actions and the things that happen to her. In her mental state examination her mood was euthymic and she had delusions of control, thought broadcasting and in her perceptions she had visual perceptual abnormality where she saw the same object she would look at in another direction but they are under her control. She also had second and third person auditory hallucinations. She was admitted to start on clozapine because her voices did not respond to any medication.

Discussion. Auditory hallucinations are the most commonly encountered type in schizophrenia with a prevalance of $70-80 \%$. This patient hears the words that she sees which has some features of reflex hallucinations, however in the latter the hallucination is not a transformation of the perception. This also has some qualities of thought echo, where just as the patient thinks she can hear them. However in this patient she cannot read the texts in her mind. Inner reading voices are where a person talks to oneself while reading, however in the subjective mind. In our patient this phenomenon also proved to be the most difficult to treat as all her other auditory hallucinations responded to Clozapine, while still this phenomenon remained.

Conclusion. This case is presented to describe the rare psychopathology in this patient in the form of auditory hallucinations

\section{Characterization of a novel CSF1R mutation causing hereditary diffuse leukoencephalopathy with spheroids in a case presenting with young-onset dementia}

Walid Nasr ${ }^{1 *}$, Mahmoud Gad ${ }^{1}$ and Tareq Qassem ${ }^{2}$

${ }^{1}$ Al-Amal Hospital, Ministry of Health and Prevention and

${ }^{2}$ Maudsley Health, Al-Amal Hospital, Ministry of Health and Prevention, Mohammed Bin Rashid University Of Medicine and Health Sciences, Institute of Psychiatry, Ain Shams University ${ }^{\star}$ Corresponding author.

doi: 10.1192/bjo.2021.345

Objective. This poster aims to report an unregistered mutation CSF1R gene in a patient presenting young-onset dementia.

Hypothesis: Novel heterozygous deletion-insertion mutation in the Colony-Stimulating Factor 1 Receptor (CSF1R) gene is linked to a case of hereditary diffuse leukoencephalopathy with spheroids (HDLS), presenting with young-onset dementia.

Background. CSF1R mediates proliferation, differentiation, and survival of monocytes/ macrophages and microglia. Pathogenic variants in the CSF1R gene cause autosomal dominant diffuse hereditary leukoencephalopathy with spheroids characterized by variable behavioural, cognitive, and motor changes, usually presenting with young-onset dementia. The average lifespan after the start of the symptoms is often 6 years.

Case report. Molecular genetic analysis of whole-exome sequencing (WES) was carried out for a 49-year-old male patient presenting with rapid cognitive decline, behavioural symptoms and impaired sphincteric control.

Discussion. WES identified the heterozygous deletion-insertion variant c.2356_2357delinsAC p.(Leu786Thr) (chr5:14943586749435868; hg19) in the CSF1R gene. To the best of our knowledge the variant has not been described in the literature so far (HGMD 2019.3). No allele frequencies in the general population have been documented.

Conclusion. We believe that we have identified a novel mutation in the CSF1R gene. This mutation is likely to be linked to this patient presenting with young-onset dementia. 